\title{
PROSPECTING FOR VOIDS WITH VERTICAL RADAR PROFILING
}

\section{L.F. IOANNIS ${ }^{1}$}

\section{ABSTRACT}

Vertical Radar Profiling measurements were conducted to image subsurface cavities encountered in Akrotiri Archaeological Excavations area on Thera Island. The vertical radar profiling technique is able to explore much deeper than conventional surface GPR because it uses wells. The transmitting-receiving antenna unit was moved within the excavated well along six vertical profile lines in equally divided positions. A local electrical resistivity survey preceded the GPR profiles to investigate if the conductivity of the pyroclastic formation satisfies the presuppositions to conduct GPR measurements. The vertical GPR profiles revealed locations where cavities exist but they were unable to show their shape and extent. Cross-well seismic tomography images supported the vertical radar profiling results.

KEY WORDS: Vertical GPR measurements, voids detection.

\section{ПЕРIАНЧн}

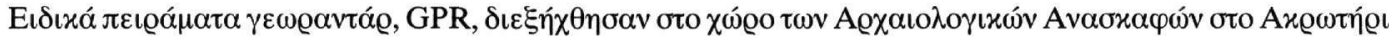

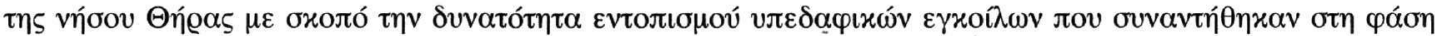

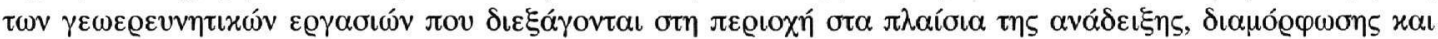

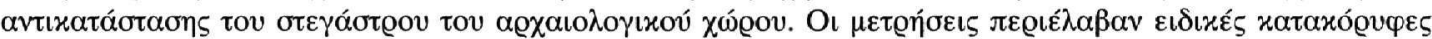

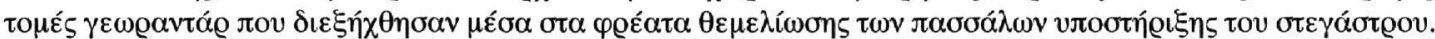

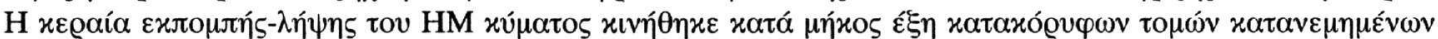

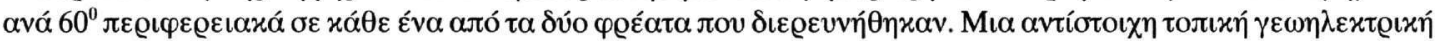

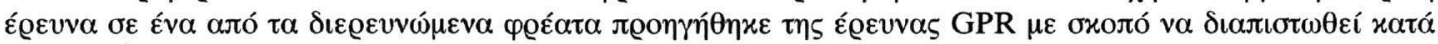

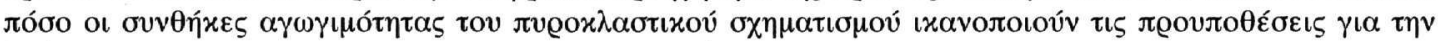

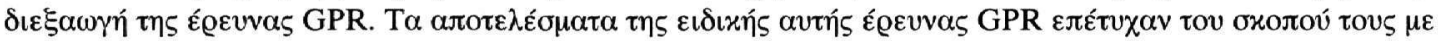

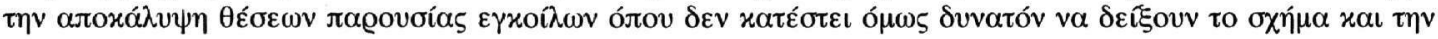

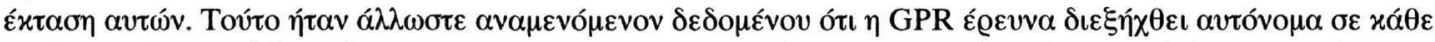

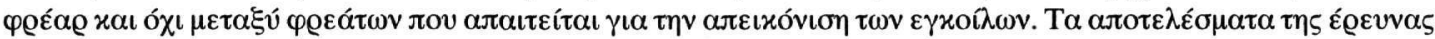

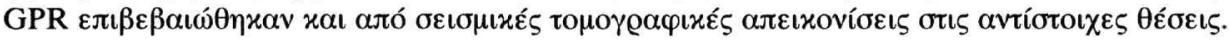

\section{INTRODUCTION}

Ground Penetrating Radar (GPR) has traditionally been successful at locating cavities, although most commonly at a large scale. In recent years it has been applied extensively to civil and environmental engineering, archaeological surveys, and many other applications.

Generally, both the transmitting and receiving antennas are located on the ground surface, so the detectable range of surface GPR is shallow because of strong wave absorption in the near-surface wet soil. Even when the soil is dry, a strong reflection interface in the shallow part returns most of the energy and only very week energy penetrates into deep region, severely limiting the detection depth. The vertical radar profiling technique is a new method that is able to explore much deeper than surface GPR by using wells (Sato, 1997; Sato et al., 1997a).

The basic idea and its advantages are in principle the same as vertical seismic profiling (VSP) for seismic measurements. When the transmitter and receiver in a well is beneath the near-surface the energy reflected by the objects back to the receiver is comparatively stronger than that of reflected waves passing through the nearsurface layer twice. In addition, the influence of noise is weaker because the transmitter and receiver in the well are free from outside noise giving thus the ability to detect much deeper features than surface GPR.

1. Geophysics \& Geothermics Dpt., University of Athens, Panepistimiopolis Ilisia 15784, Greece. E-mail: jlouis@geol.uoa.gr 
In this paper, we show an example (Louis, 2001) of vertical radar profiling applied to Akrotiri archaeological excavation area on Thera island aiming to detect underground cavities and to provide some information in the framework for replacing the existing old roof of the archaeological excavation area.

\section{THE HISTORY}

Akrotiri archaeological site is one of the most important prehistoric settlements of the Aegean. The first habitation at the site dates from the Late Neolithic times (at least the 4th millennium B.C.). During the Early Bronze Age (3rd millennium B.C.), a sizeable settlement was founded and in the Middle and early Late Bronze Age (ca. 20th-17th centuries B.C.) it was extended and gradually developed into one of the main urban centres and ports of the Aegean. The large extent of the settlement (ca. 20 hectares), the elaborate drainage system, the sophisticated multi-storeyed buildings with the magnificent wall paintings, furniture and vessels, show its great development and prosperity. ("Akrotiri of Thera", Hellenic Ministry of Culture http://www.culture.gr/2/21/211/ 21121a/e211ua08.html)

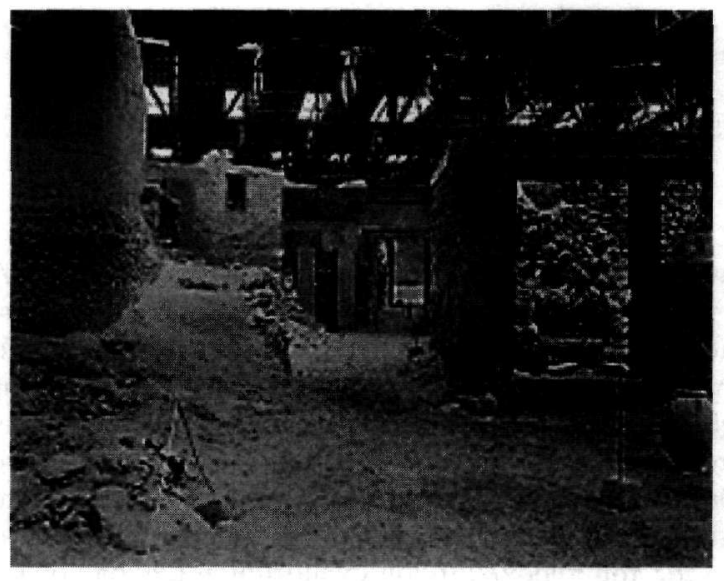

The town's life came to an abrupt end in the last quarter of the 17th century B.C. when the inhabitants were obliged to abandon it as a result of severe earthquakes. The eruption followed. The volcanic materials covered the entire island and the town itself. These materials, however, have protected up to date the buildings and their contents, just like in Pompeii.

Systematic excavations were begun in 1967 by Professor Sp. Marinatos under the auspices of the Archaeological Society at Athens. He decided to excavate at Akrotiri in the hope of verifying an old theory of his, that the eruption of the Thera volcano was responsible for the collapse of the Minoan civilization. Since his death in 1974, the excavations have been continued under the direction of Professor Ch. Doumas.

\section{THE EXPERIMENT}

In Akrotiri archaeological excavation area the Archaeological Society at Athens operates a project where the existing old roof in the Prehistoric Minoan settlement (figures 1 and 2) is going to be replaced with a new modern one. The roof under construction (100X159 sq. meters area) will be supported by pillars embedded in an equal number of wells filled with concrete. 


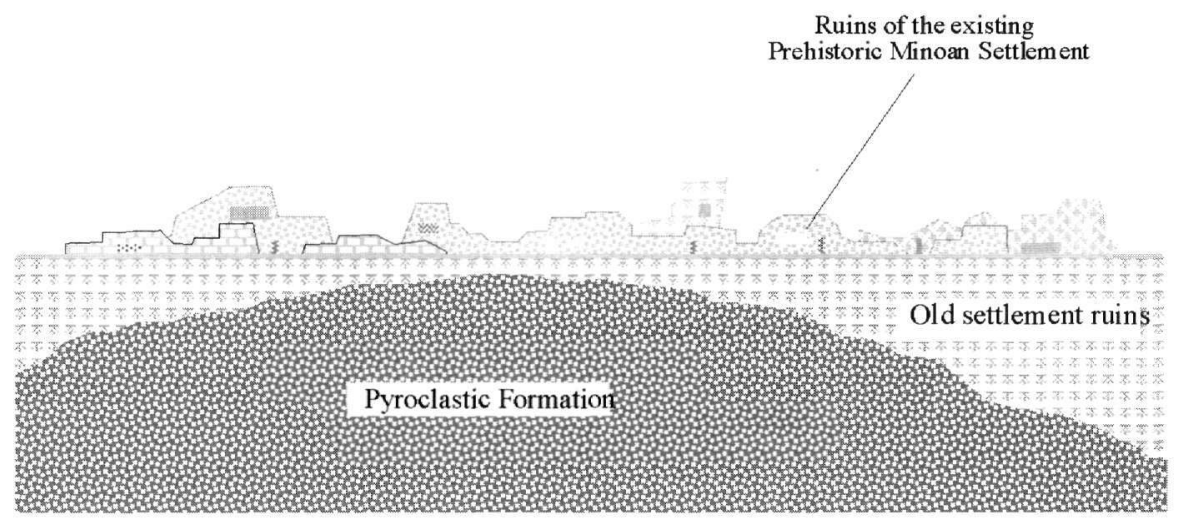

Figure 1. Sketch diagram of the Prehistoric Minoan settlement ruins as they appear today after the archaeological excavations.

The foundation wells (1.2 meters in diameter and 8 meters depth) are open up in the pyroclastic volcanic formation basement rock. The ceiling of the pyroclastic formation is met at depths varying between 2 and 15 meters below the ground surface where the Prehistoric Minoan settlement is situated (figure 2). The layer between ground surface and pyroclastic formation consists of ruins of an older town destroyed completely by a large earthquake.

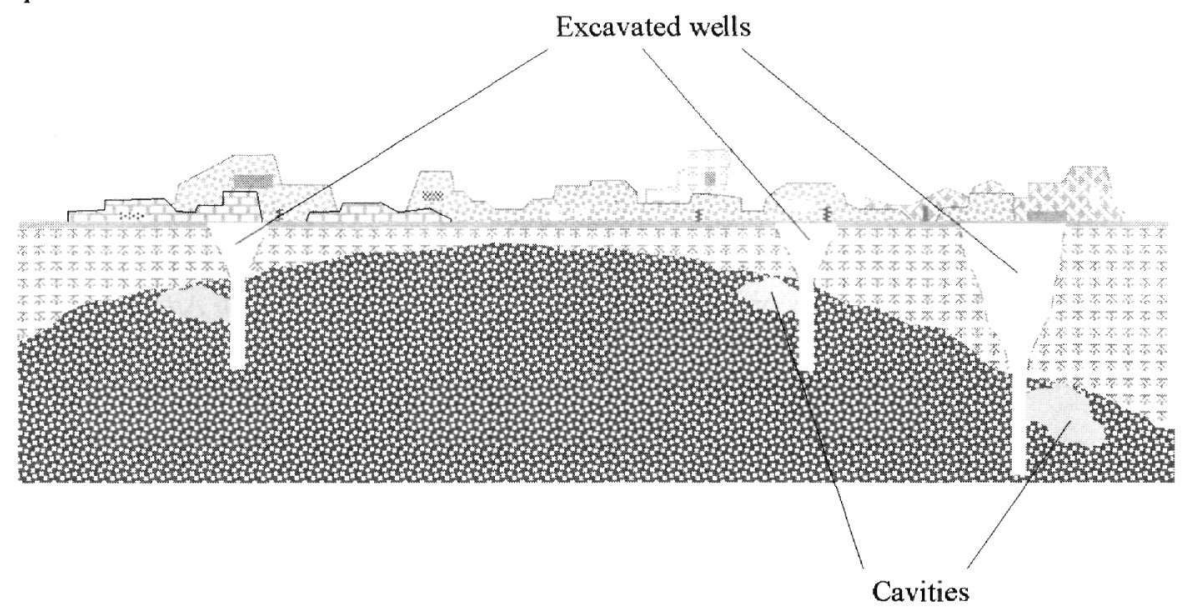

Figure 2. Sketch diagram of the excavated wells with cavities in their walls.

Man-made cavities appeared in the walls of some foundation wells (figure 2) during the excavations. Some of them were void while others were filled with stones, ceramics etc. According to the new findings from the excavations they probably concern prehistoric tombs.

Since the target of prospect is buried under a thick layer of ruins it was evident that surface GPR surveys would be incapable of detecting voids superimposed by such complex structures. This is due to the limitations, inherent in the physics of the method, where the receiver picks up all the returns such as large reflections from the ground surface and from many objects in different positions. Consequently any, later in time, received signal may be composed of the sum of returns from many objects in different positions attenuating thus the signal from the target.

The existence of the excavated wells was an excellent opportunity for us to apply the vertical profiling technique inside the well. 
Six GPR traverses (profiles) L1 to L6 were conducted in each well along the vertical axis one every $60^{\circ}$ (figures 3 and 4). Each GPR profile run in two directions: from top to bottom and bottom to top. The first $1 \mathrm{~m}$ length of the well was not investigated due to the presence of a metal ring situated at the top of the pyroclastic formation to support the excavated loose material. A wooden plank $7 \mathrm{~m}$ long was inserted between the antenna and the borehole wall to achieve thus a smooth motion of the antenna unit. Seven markers, $1 \mathrm{~m}$ apart, marked on the wooden plank served to register a respective marker on the GPR records when the moving antenna was crossing them.

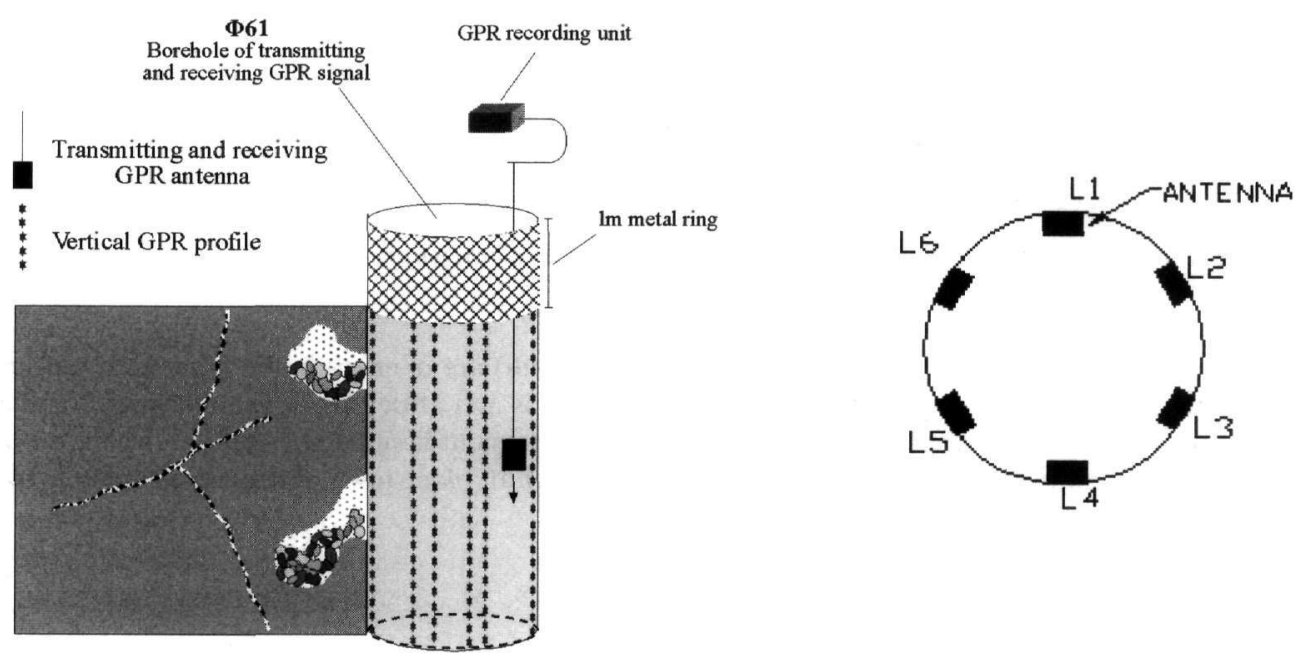

Figure 3. Field layout with the vertical GPR profiles on the walls of the borehole.
Figure 4. Horizontal cross section of the borehole with the positions of the 6 vertical GPR profiles.

The GPR unit used is a SIR SYSTEM 2000 of GSSI (Geophysical Survey Systems Inc.) It contains the system generating EM impulse, an array processor for simple data processing and an A/D acquisition board for real time digital recording in the internal hard disk. A $400 \mathrm{MHz}$ antenna was used for transmitting the electromagnetic energy and receiving back the echoes.

An electric winch was used to drive the operator of the antenna unit down to the wall (figures 5 and 6 ). Since the top of the pyroclastic material is met 10 to 15 meters below the ground surface, he had to cross these depths in order to arrive at the beginning of the GPR profile. Then he had to move another $8 \mathrm{~m}$ downwards along the vertical profile line.

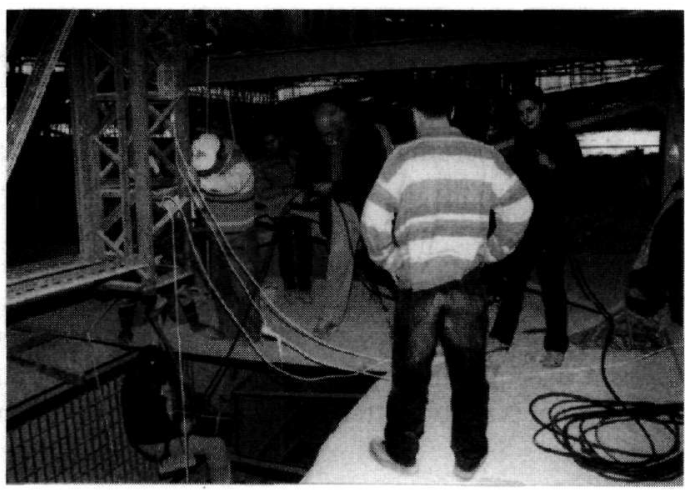

Figure 5. The operator, attached with the antenna unit, at the beginning of his journey.

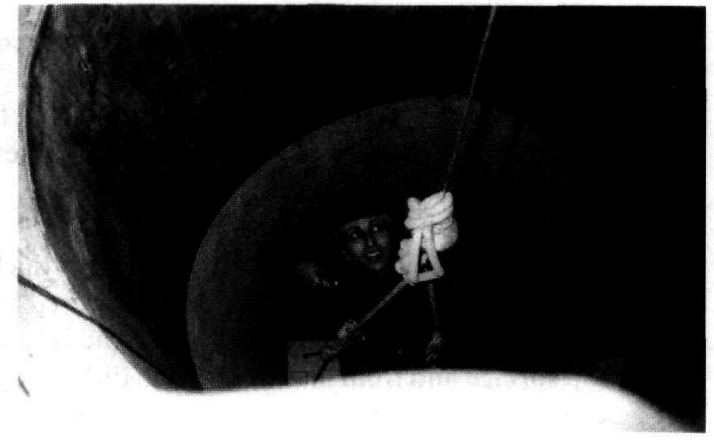

Figure 6. The operator at the top of the pyroclastic material ready to start GPR measurements another $8 m$ downwards. 
The control unit generates a trigger pulse signal to the transducers transmitting section. A short burst of electromagnetic energy is then radiated to the underground where it travels at a velocity that depends on the dielectric constant of the medium. The energy reflected back by the interfaces between media with different dielectric constants is received by the receiving section of the transducer and sends it to the control unit. An improvement of the signal/noise ratio was obtained by the application of specific digital horizontal and vertical filters.

A local electrical resistivity survey preceded the GPR profiles. The resistivity measurements were conducted on the vertical walls of $\Phi 61$ well aiming to investigate the conductivity of the pyroclastic formation. This was considered as absolutely necessary since it is well known that the moisture content of different regions is the dominant factor in influencing both the conductivity and dielectric constant and hence the attenuation and velocity of the transmitted EM wave.

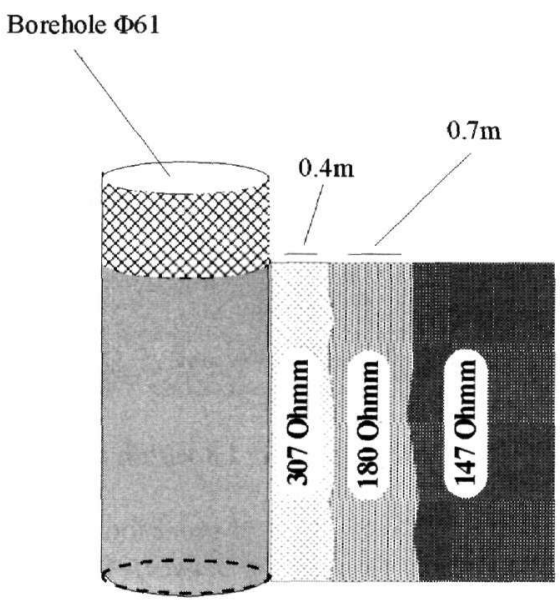

Figure 7. Horizontal resistivity variation in well $\Phi 61$ attributed to the different moisture content in the pyroclastic formation.

Figure 7 shows the results of the resistivity experiment. We can see that the electrical resistivity of the pyroclastic formation is laterally decreasing. The outer thin layer of the formation exhibits almost high resistivity (307 Ohmm) due to its dry condition. Moving deeper, in the horizontal direction, the resistivity is gradually decreasing due to the higher moisture content of the formation. Summarizing we can say, in general, that the condition of the formation from the conductivity distribution point of view rather satisfies the presuppositions to conduct a GPR survey.

\section{DATA PROCESSING AND RESULTS}

The GPR records obtained in the field were of good quality. The digitally recorded data were processed using RADAN III software.

The interpretation was focused to recognize the different electromagnetic anomalies and their geometrical relationships and shapes.

The difficulty in this case is that we are searching for voids filled with a material having the same electromagnetic properties with the main rock. In that case the discrimination is difficult since GPR receives reflections from the interface of two bodies having different electromagnetic properties. The electromagnetic transmission depends on the dielectric constant of the media and the most important factor affecting it is the moisture content. 


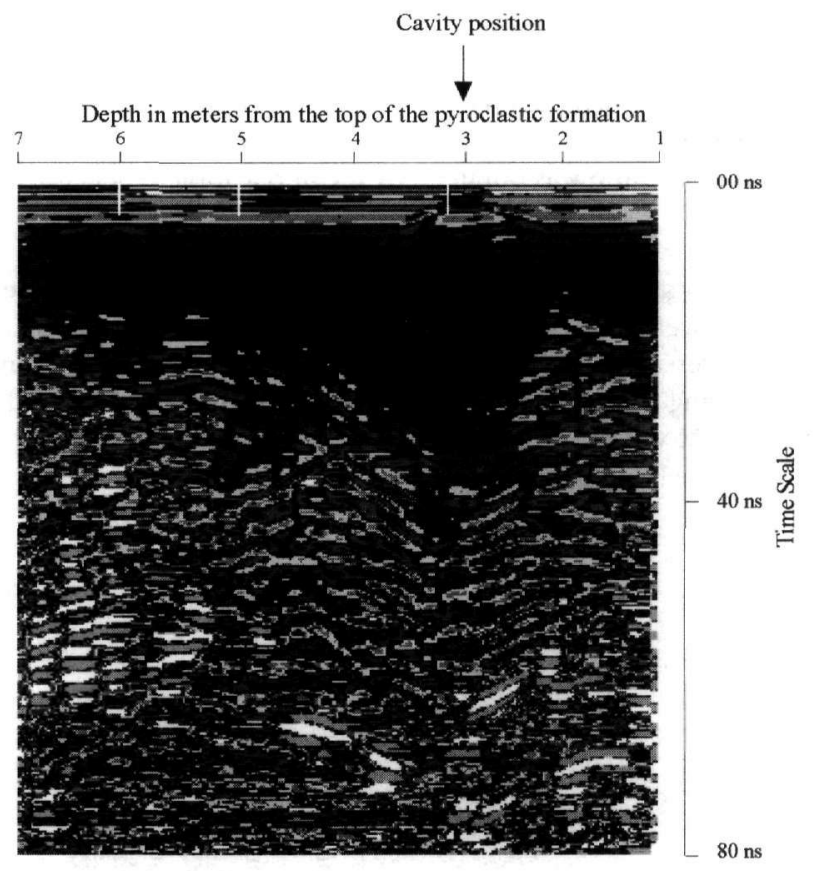

Figure 8. GPR profile line L3 within the well $\Phi 39$.

A major limitation of the method remains the lack of precision concerning the determination of the wave propagation velocity in relation to the depth. Correlation between GPR data and a known target enables timedepth calibration. Figure 8 shows the record of the GPR line L3 obtained within the well $\Phi 39$. An anomaly is visible between depths 2.8 and 3.2 meters, which corresponds to a cavity revealed during the excavation. The dark area from 3 to $5 \mathrm{~m}$ probably depends on the low resistivity of the ground caused from the different moisture content in it.

Figure 9 shows the record of GPR line L6 obtained within the well $\Phi 61$. The hyperbola pattern visible at the depth of 6 meters was attributed to a void. This profile was processed in order to obtain a better resolution. Figure 10 shows the same record after the application of a horizontal and vertical filter and Hilbert transform. The hyperbola pattern visible at the depth of 6 meters is now clear. In the same figure a new hyperbola pattern is now visible at the depth of the 3 meters. 


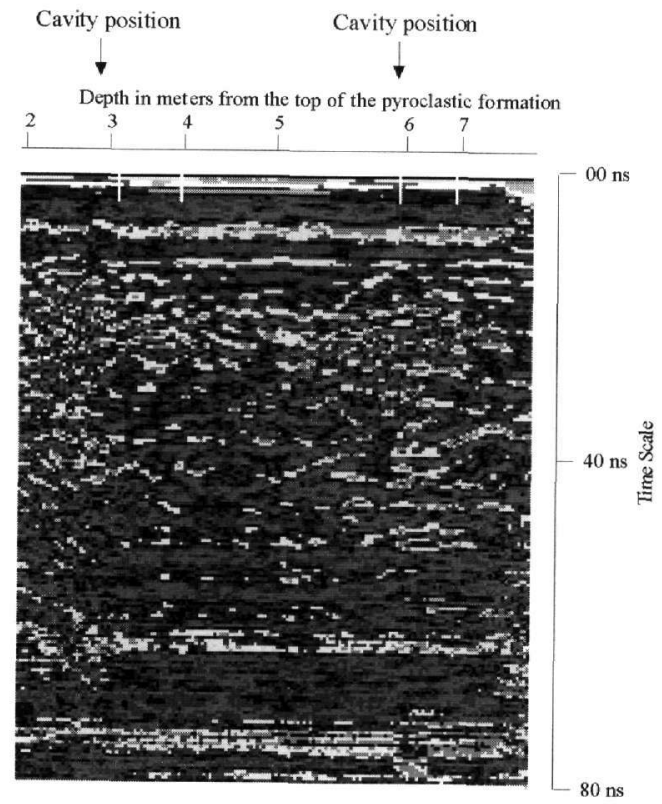

Figure 9. GPR profile line L6 within the well $\Phi 61$ (Deconvolution).

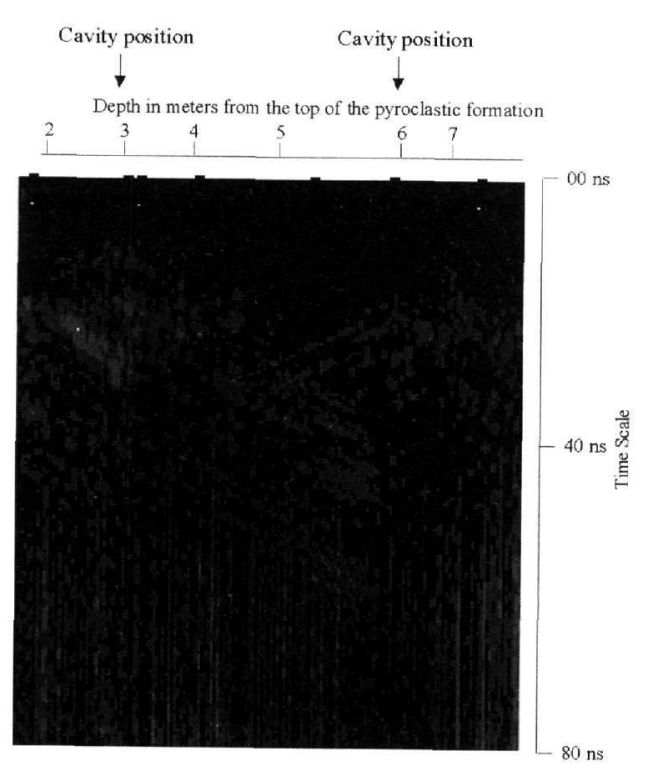

Figure 10. GPR profile line L6 within the well $\$ 61$ (Horizontal filter and Hilbert transform).

Figure 11 shows a cross-well seismic tomography image of the area adjacent to the same well $\Phi 61$. It is very clear that two low seismic velocity anomalies occur at the same depths. This seismic image supports our GPR results concerning the investigated well.

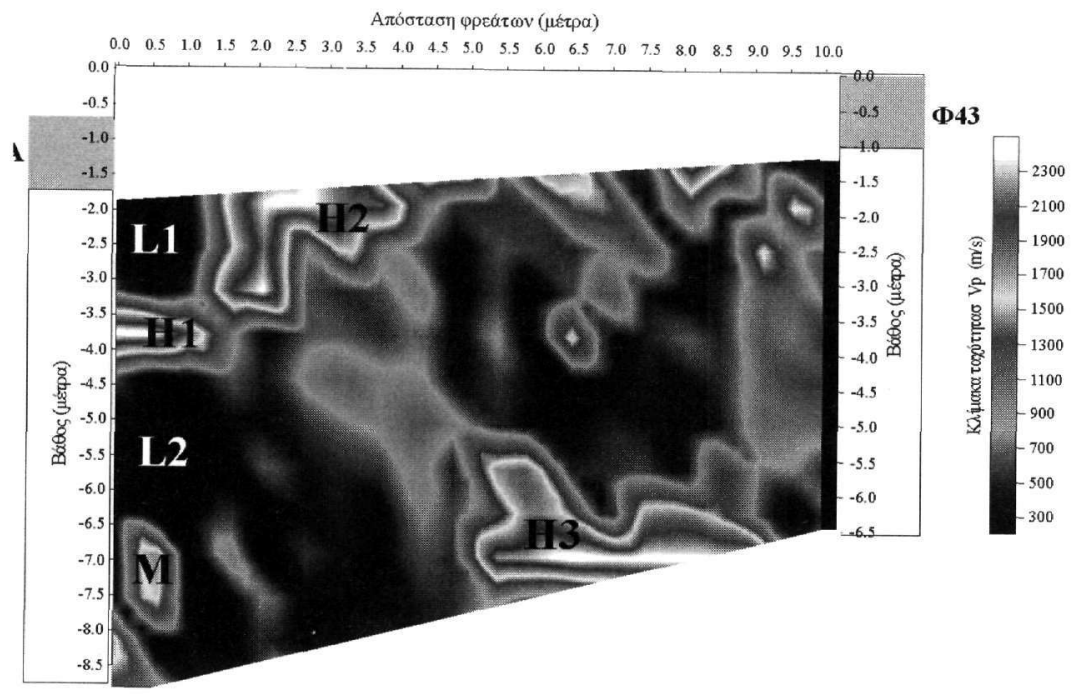

Figure 11. Seismic velocity image obtained from cross-well seismic tomography experiment carried out in the same well. 


\section{CONCLUSIONS}

The vertical radar profiling method enables to explore much deeper than surface GPR by using wells. This is caused since the transmitting and receiving antennas are located within the well so the strong wave absorption observed in the near-surface GPR surveys is almost removed.

The vertical GPR profiles revealed locations where cavities exist but they were unable to show their shape and extent. Cross-well seismic tomography images supported the vertical radar profiling results.

\section{REFERENCES}

"Akrotiri of Thera", http://www.culture.gr/2/21/211/21121a/e211ua08.html Hellenic Ministry of Culture.

SATO, M., LU, Y., AND NITSUMA, H., 1997, Subsurface imaging by vertical radar profiling and its application to Tajiri archaeological site: Butsuri-Tansa, 50, 196-207.

SATO, M., 1997a, Vertical radar profiling for pipe detection: Internat. Symp. Engin. Environ. Geophys. Proceedings, 308-313.

ZENG, X., AND MACMECHAN, G. A., 1997, GPR characterization of buried tanks and pipes: Geophysics, 62 , 797-807.

LOUIS, F. I., 2001, Geophysical Investigations within the frameworks of the exhibition and replacement of the old roof in the archaeological excavations area at Akrotiri on Thera island: Technical Report submitted to the Archaeological Society at Athens. 